\title{
Ellipsometry Analytical Complex for Measuring near the Surface Plasmon Resonance in Colorectal Cancer Diagnosis
}

\author{
DOI: 10.17691/stm2019.11.2.10
}

Received March 1, 2018

V.N. Kruchinin, PhD, Senior Researcher, Laboratory of Ellipsometry';

M.V. Kruchinina, MD, DSc, Leading Researcher, Laboratory of Gastroenterology2;

Associate Professor, Department of Propedeutics of Internal Diseases ${ }^{3}$;

Ya.I. Prudnikova, PhD Student, Laboratory of Gastroenterology2;

E.V. Spesivtsev, PhD, Senior Researcher, Laboratory of Ellipsometry';

S.V. Rykhlitskiy, PhD, Head of the Laboratory of Ellipsometry ${ }^{1}$;

S.E. Peltek, PhD, Head of the Laboratory of Molecular Biotechnology4;

S.V. Shehovtsov, PhD, Senior Researcher, Laboratory of Molecular Biotechnology4;

G.V. Shuvalov, PhD, Director ${ }^{5}$

${ }^{1}$ Rzhanov Institute of Semiconductor Physics, Siberian Branch of the Russian Academy of Sciences,

13 Akademika Lavrentieva Avenue, Novosibirsk, 630090, Russia;

${ }^{2}$ Institution of Internal and Preventive Medicine - Branch of the Federal Research Center Institute

of Cytology and Genetics, Siberian Branch of the Russian Academy of Sciences, 175/1 Borisa Bogatkova St.,

Novosibirsk, 630089, Russia;

${ }^{3}$ Novosibirsk State Medical University, 52 Krasny Prospect, Novosibirsk, 630091, Russia;

${ }^{4}$ The Federal Research Center Institute of Cytology and Genetics, Siberian Branch

of the Russian Academy of Sciences, 10 Akademika Lavrentieva Avenue, Novosibirsk, 630090, Russia;

${ }^{5}$ Siberian State Order of the Red Banner of Labor Research Institute of Metrology, 4 Prospekt Dimitrova,

Novosibirsk, 630004, Russia

The aim of the study was to assess the ellipsometry analytical complex for its diagnostic potential related to colorectal cancer and metastases of different localization. The proposed ellipsometry was aimed to complement the current non-invasive optical methods based on the reflection of polarized light near the surface plasmon resonance under full internal reflection.

Materials and Methods. We measured the highly specific and sensitive reaction between tumor-associated M2-pyruvate kinase and the respective monoclonal antibodies immobilized on chips. The biological material (blood serum) was obtained from healthy (cancer-free) subjects $(n=19)$ and from patients with colorectal cancer of various localizations $(n=49)$. The examined patients were divided into three subgroups depending on localization of the metastases: $1^{\text {st }}$ subgroup - local-regional colorectal cancer $(n=15) ; 2^{\text {nd }}$ subgroup - hepatic metastases only $(n=18) ; 3^{\text {rd }}$ subgroup - extrahepatic metastases $(n=16)$. We also determined the concentrations of tumor M2-pyruvate kinase during its interaction with the monoclonal antibodies. In this, we measured the refractive index of the chip near-surface area by using highly sensitive optical systems able to measure the light polarization near the surface plasmon resonance.

Results. The interaction of serum M2-pyruvate kinase with the antibodies leads to a noticeable change in the optical properties of the medium, which indicates a high specificity of this reaction. We found significant differences (>20-fold) in the equilibrium levels of serum M2-pyruvate kinase both in healthy individuals and in patients with colorectal cancer, as well as in patients with metastases of various localizations. The diagnostic potential of these optical methods in respect to cancer-specific serum M2-pyruvate kinase as a marker of colorectal cancer was confirmed by subsequent ROC analysis.

Conclusion. The results obtained are promising for the development of methods for early diagnosis of colorectal cancer, for the detection of metastases of various locations, and (in the long term) for the detection of the recurrent disease, as well as for the treatment follow-up.

Key words: cancer diagnostics; colorectal cancer; ellipsometry; surface plasmon resonance; tumor M2-pyruvate kinase.

Corresponding author: Vladimir N. Kruchinin, e-mail: vladd.kruch@yandex.ru 


\section{Introduction}

Colon cancer is the third most common cancer among men and the second one - among women $[1,2]$. Despite a detailed preoperative examination of patients with colorectal cancer (CRC) including hightech methods such as computed tomography (CT) [3], in some cases, distant metastases may go undetected. In the existing protocols, a histological examination of the surgically removed materials is used to determine the depth of the tumor and the involvement of lymph nodes; the histological criteria are decisive for determining the stage of the disease. The American Society of Clinical Oncology [4] recommended annual examinations including CT and the cancer-fetal antigen test every three months for three years for patients with stage II and III cancer, if they are scheduled for surgical intervention or chemotherapy due to metastases [5]. Intensive postoperative observation is crucial for the detection of resectable metastases $[6,7]$. Therefore, the search continues for early markers indicative of the stage of cancer and/or the presence of metastases.

Pyruvate kinase, a key enzyme of glucose metabolism, exists in various isoforms which are tissuespecific. Type $L$ pyruvate kinase is found in the liver and proximal renal tubules, type $\mathrm{R}$ - in red blood cells, type M1 - in muscles and the brain, and type M2 - in the lungs. The active forms of all these isoenzymes consist of four identical subunits (i.e., they are all tetramers) [8, 9]. In tumor cells, the variety of tissue-specific isozymes shrinks, and the M2-pyruvate kinase (M2-PK) becomes the dominant form. As the amount of M2-PK increases, this isozyme switches from a tetramer to a dimer, which has a lower enzymatic activity.

The dimeric form of M2-PK characteristic of tumor cells is called tumor M2-PK. Dimerization of tumor M2PK occurs due to phosphorylation or direct binding of oncoproteins and leads to a decrease in the enzyme activity, similar to the role of glucose in the synthesis of nucleic acids, phospholipids, and amino acids. Tumor M2-PK refers to the family of biomarkers indicative of the presence of tumor in the body [10-13]. If there is a malignant process, the blood plasma level of this dimeric M2-PK increases significantly. Thus, the determination of tumor M2-PK in the serum of patients with CRC is substantiated because it reflects the specific tumor metabolism [14].

Measuring the levels of biomarkers is often difficult because of their low concentrations in body tissues; it is, therefore, important to improve the techniques capable of detecting very low concentrations of metabolites and increase the sensitivity of these assays. Identifying low levels of M2-PK in the serum is promising for the early diagnosis of $\mathrm{CRC}$, the detection of occult metastases before or after surgery, and the optimization of the CRC staging. All those are key factors that determine the treatment strategy.

Among the currently used methods for the early diagnosis of oncological diseases, there are optical methods based on the analysis of light reflected, transmitted or scattered by biological tissues; most of them are minimally invasive or non-invasive. One of those is based on the measurement of reflected polarized light near the surface plasmon resonance (SPR) with full internal reflection [15-17]. This technique is highly sensitive to changes in the surface and the near-surface area of the resonant medium; for example, metal films (gold) on the surface of a solution (blood serum). In this example, the light is reflected from the surface of a glass plate covered with a thin layer of gold (chip) with monoclonal antibodies specific to tumor M2-PK, immobilized on the chip's surface. To measure the reflected polarized light near the point of SPR, the method of ellipsometry is increasingly used. It allows one to measure the reflected polarized light with high accuracy, determine the optical characteristics of the reflecting object [18], and evaluate the number of reactive biomolecules.

The aim of the study is to develop a technique for increasing the sensitivity of the current minimally invasive optical methods and improve their potential for the diagnosis of colorectal cancer with variously located metastases.

To that end, we used spectral ellipsometry near the SPR to measure the reaction between tumor M2-PK from patients' serum and specific monoclonal antibodies to this enzyme [19].

\section{Materials and Methods}

In this study, 68 people were examined: 19 conditionally healthy individuals (average age $-53 \pm 8$ years) with no cancer or other manifesting disease, and 49 patients (average age $-52 \pm 9$ years, 22 women and 27 men) with CRC of various localizations. The locations included: the transverse colon $(n=3)$, the descending colon $(n=3)$, the sigmoid colon $(n=9)$, the rectosigmoid $(n=6)$, the rectum $(n=22)$, as well as primary multiple colon tumors $(n=6)$. Histologically, all patients were diagnosed with adenocarcinoma of various grades of differentiation, mostly moderate. The diagnoses were verified by comprehensive clinical and instrumental examinations (ultrasound, CT, MSCT, colonoscopy, fibrogastroduodenoscopy, and histological analysis) in a specialized clinic for cancer patients.

Patients with CRC were divided into three subgroups depending on the location of the metastases: the $1^{\text {st }}$ subgroup included 15 patients with local-regional CRC (7 of them had II, 8 - III stage of the disease); the $2^{\text {nd }}$ subgroup consisted of 18 patients with metastases limited to the liver (12 of them had single metastases, the rest had multiple metastases with sizes varied from 16 to $92 \mathrm{~mm}$ ); the $3^{\text {rd }}$ subgroup included 16 patients with extrahepatic metastases (supraclavicular lymph nodes, lungs, bones, brain). The patients of the $2^{\text {nd }}$ and $3^{\text {rd }}$ subgroups were confirmed as having stage IV CRC. 
Three patients (20\%) from the $1^{\text {st }}, 6(33 \%)$ from the $2^{\text {nd }}$, and $11(69 \%)$ from the $3^{\text {rd }}$ subgroups underwent adjuvant polychemotherapy for 3 months.

The study was carried out in accordance with the Helsinki Declaration (2013) and approved by the Committee of Biomedical Ethics of the Research Institute of Therapy and Preventive Medicine affiliated with the Institute of Cytology and Genetics of the Siberian Branch of the Russian Academy of Sciences. All patients signed informed consent to participate in the study.

We used blood serum obtained by centrifuging (2000 rpm for $20 \mathrm{~min}$ ) samples of whole blood taken at fast. For further research, the serum was diluted with phosphate buffer. The paper presents the data obtained by diluting the serum samples 1:250.

Highly specific monoclonal Anti-PKM2 antibodies [EPR10138 (B)] (Abcam, USA) were immobilized on the gold surface $(\sim 30-40 \mathrm{~nm})$ according to the procedure described in [20]. A thin film of gold was used as a resonant medium and was part of the glass plates used in the subsequent ellipsometry measurements (Ellips- SPEC; ISP SB RAS, Russia) [21]. The gold layer was also a component of special chips used for the measurements by the optical biosensor ProteOn XPR36 system (Bio-Rad, USA). The inner side of the plates for ellipsometry measurements was fixed in a special flow cell made according to the Krechman design (Figure 1) [22]. The sample (serum) was injected into the phosphate buffer solution that continuously circulated through the cell. A prism was attached to the reverse side of the plates (through a layer of glycerin); this was used for the ellipsometry measurements. The two parameters measured in ellipsometry (angles $\Psi$ and $\Delta$, i.e., the ratio of the amplitude and the phase difference of the reflected and incident beams of polarized light, respectively) are related to the parameters of the reflecting system by the equation

$$
\operatorname{tg} \Psi \cdot e^{i \Delta}=R_{p} / R_{s}
$$

where $R_{p}, R_{s}$ are the complex light reflection coefficients for the waves polarized in the incident plane and perpendicular to it; the coefficients depend on the optical properties of the reflecting system [23].

The SPR is observed if:

$$
\sqrt{\varepsilon_{p r}} \cdot \sin \left(\phi_{0}\right)=\sqrt{\frac{\varepsilon_{m e t} \cdot \varepsilon_{s o l}}{\varepsilon_{m e t}+\varepsilon_{s o l}}},
$$

where $\varepsilon_{p r}, \varepsilon_{m e t}, \varepsilon_{s o l}$ are dielectric constants of the prism/ plate material, the metal layer (gold) and the solution (serum in phosphate buffer) [22].

When the SPR is reached (equation 2), any change in the dielectric constant of the solution, will lead to a shift in the SPR absorption peak and, subsequently, to a change in the ellipsometric angles $\Psi$ and $\Delta$ (equation 1). It has been experimentally confirmed that ellipsometric measurement made under the conditions of resonant light absorption is 2-3 orders of magnitude more

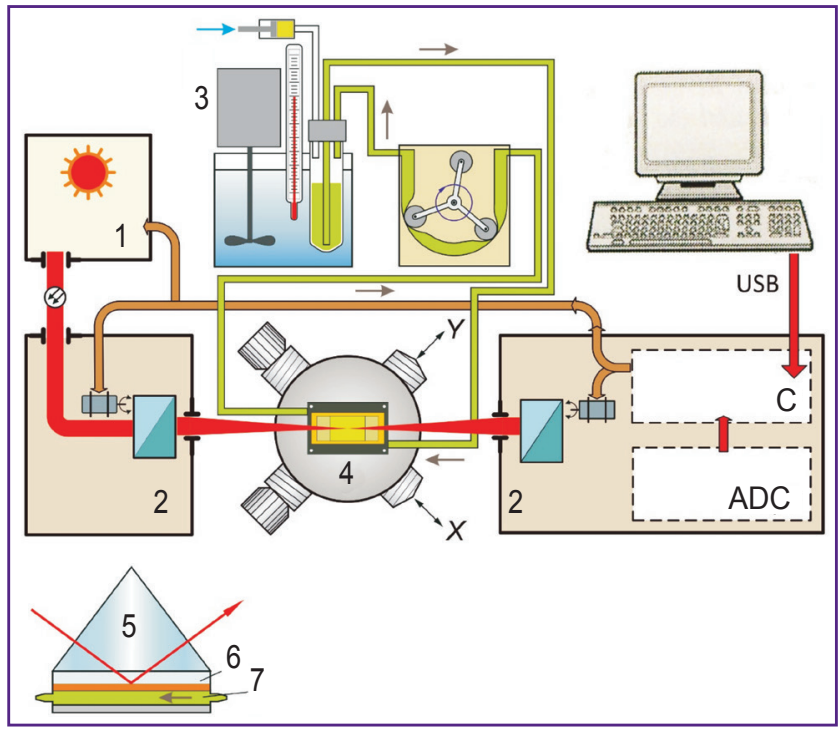

Figure 1. Ellipsometry analytical complex with the surface plasmon resonance:

1 - monochromatic light source; 2 - ellipsometer; 3 - the system for sample inlet, temperature control, and circulation; 4 - the precision object stage with SPR cell. In the bottom part of the Figure, the SPR cell is schematized: 5 - prism; 6 - gold-coated glass plate; 7 - cell housing. Here, ADC is an analog-to-digital converter, $\mathrm{C}$ is a controller

sensitive and capable of detecting individual protein molecules at concentrations of tens nanogram per milliliter.

In parallel to the above technique, we also used the optical biosensor ProteOn XPR36 system to follow the reaction between specific monoclonal antibodies to M2-PK and blood serum samples from healthy people and patients with CRC. The ProteOn XPR36 system is also based on the measurement of reflected polarized light under the conditions of SPR. Antibodies to human tumor M2-PK were covalently linked to the gold-coated surface of the GLC sensor chip. In both techniques, the measurements were made when the SPR was reached and observed. The different results obtained with the Ellips-SPEC ellipsometer vs the ProteOn XPR36 system could be due to the difference in the parameters determined in these two methods (in the ellipsometry, the phase component of the signal is also taken into account).

\section{Results and Discussion}

In our study, samples of the blood serum obtained from healthy subjects and patients with CRC were used. Figure 2 shows the kinetics of the reaction between monoclonal antibodies to tumor M2-PK and the respective serum antigens when determined either by the spectral ellipsometry and by the optical biosensor ProteOn XPR36 system (in both cases, according to the "near SPR" principle). 


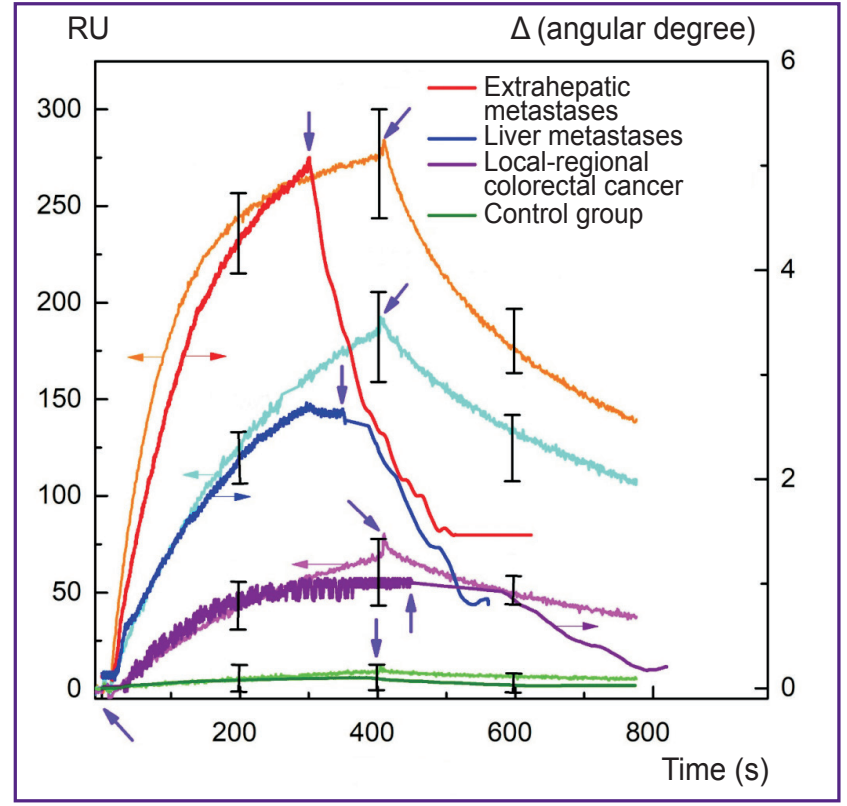

Figure 2. Sensograms reflecting the binding and disintegration of immuno-complexes between the serum antigens and the specific monoclonal antibodies to tumor M2-PK in patients with colorectal cancer and healthy individuals (sera samples diluted 1:250)

The arrows indicate the moments of sample additions and washings. In the pairs: darker curves - Ellipse-SPEC data, lighter curves - ProteOn XPR36 data

The equilibrium levels of serum M2-PK as determined using the surface plasmon resonance $(\mathrm{M} \pm \mathrm{m})$

\begin{tabular}{|c|c|c|}
\hline Groups of subjects & $\begin{array}{l}\text { Average level } \\
\text { of serum M2-PK } \\
\text { (RU) }\end{array}$ & $p$ less than \\
\hline Group of comparison ( $n=19)$ & $11.6 \pm 4.5$ & - \\
\hline $\begin{array}{l}1^{\text {st }} \text { subgroup, local-regional colorectal } \\
\text { cancer }(n=15)\end{array}$ & $67.4 \pm 9.8$ & $10^{-7 *}$ \\
\hline $\begin{array}{l}2^{\text {nd }} \text { subgroup, stage IV colorectal } \\
\text { cancer with liver metastases }(n=18)\end{array}$ & $162.5 \pm 17.8$ & $\begin{array}{c}10^{-7 *} \\
1-2=10^{-6}\end{array}$ \\
\hline $\begin{array}{l}3^{\text {rd }} \text { subgroup, stage IV colorectal } \\
\text { cancer with extrahepatic metastases } \\
(n=16)\end{array}$ & $269.2 \pm 14.6$ & $\begin{array}{c}10^{-7 *} \\
1-3=10^{-7} \\
2-3=5.1 \cdot 10^{-6}\end{array}$ \\
\hline
\end{tabular}

* Significantly different from the comparison group; 1-2, 1-3, 2-3 - between the groups.

The equilibrium level of serum tumor M2-PK in healthy subjects was $11.6 \pm 4.5 \mathrm{RU}$ (resonance unit); it did not correlate with gender $(p=0.51)$ or age $(p=0.61)$. Per definition, $1 \mathrm{RU}$ is equivalent to the shift caused by the binding of $1 \mathrm{pg}\left(10^{-15} \mathrm{~kg}\right)$ of protein per $1 \mathrm{~mm}^{2}$ of sensitive surface and corresponds to $\sim 1.12$ angular min of ellipsometry angle $\Delta$.

The equilibrium level of tumor M2-PK in the sera of patients with local-regional CRC (1 ${ }^{\text {st }}$ subgroup) was $67.4 \pm 9.8 \mathrm{RU}$, in patients with $\mathrm{CRC}$ and liver metastases ( $2^{\text {nd }}$ subgroup) $-162.5 \pm 17.8 \mathrm{RU}$, and in patients with extrahepatic metastases ( $3^{\text {rd }}$ subgroup) $269.2 \pm 14.6 \mathrm{RU}(\mathrm{p}<0.001-0.05)$, which were manifold higher than that in non-CRC subjects (see the Table).

The results obtained with the spectral ellipsometry SPR complex "Ellips-SPEC" (see Figure 2) were comparable with those obtained with the ProteOn XPR36 system.

It was reported that levels of tumor pyruvate kinase differed at different stages of CRC [10] and that these levels were associated with the CRC stages as defined by Duke [24].

The role of tumor M2-PK in the development of metastases is intriguing. The study [25] demonstrated the involvement of tumor M2-PK in the development of new blood vessels around a wound surface; the mechanism is associated with increased secretion of vascular endothelial growth factor. With tumor growth and progression, high levels of M2-PK may ensure the formation of new vessels in the growing mass of the tumor. Thus, the tumor cells survive in an unfavorable metabolic environment and then give rise to metastasis.

The difference in the serum level of tumor M2-PK between patients with CRC and healthy individuals was determined with a sensitivity of $98 \%$, which was consistent with the results of the study [26] and exceeded the sensitivity of such methods as colonoscopy, occult fecal blood tests, levels of cancer-embryonic antigen, and the fecal M2-PK [27, 28]. In the routine laboratory practice, the fecal M2-PK is determined using monoclonal antibodies specific to the dimeric form (immuno-enzyme analysis). At a discriminant level

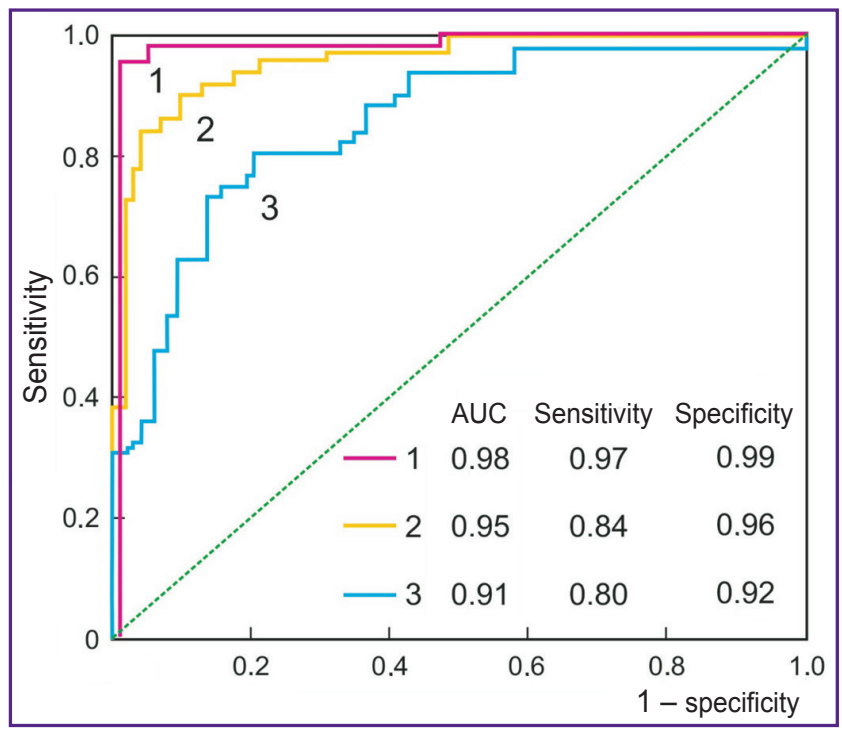

Figure 3. ROC curves for serum tumor M2-PK used for the identification of patients with colorectal cancer metastases of different localizations:

1 - patients with local-regional colorectal cancer vs healthy subjects; 2 - patients with liver metastases vs patients with local-regional colorectal cancer; 3 - patients with extrahepatic metastases vs patients with local-regional colorectal cancer 
of $4 \mathrm{U} / \mathrm{ml}$, the test specificity for CRC is $79 \%$ and the sensitivity - 85\% (with or without bleeding) [29], which is lower than the RU values obtained in the present study.

When the threshold value of serum tumor M2-PK was set at $11.6 \pm 4.5 \mathrm{RU}$, not a single case of $\mathrm{CRC}$ was missed; therefore, $43.4 \%$ of colonoscopy examinations could be avoided. The diagnostic sensitivity of ellipsometry in all examined cases of CRC increased with a decrease in the threshold values set for serum M2-PK levels.

A ROC analysis related to the confirmed CRC metastatic diseases showed the AUC values for serum M2-PK to be $0.91-0.98$ (95\% confidence interval) with rather high levels of sensitivity and specificity (Figure 3 ).

\section{Conclusion}

Using the spectral ellipsometry method and optical biosensor ProteOn XPR36 system, based on measuring light polarization near the surface plasmon resonance, we investigated the reaction between monoclonal antibodies immobilized on the chip surface, and M2pyruvate kinase from the blood serum of patients with colorectal cancer with metastases of various localizations.

Significant differences in the levels of serum M2pyruvate kinase were found in healthy individuals and patients with colorectal cancer (more than 20fold) including patients with metastases of various localizations. The diagnostic value of these optical methods in distinguishing between these groups is confirmed by the subsequent ROC analysis (AUC 0.91-0.98).

These results are promising for the development of methods for early detection of cancer, including colorectal cancer, for the detection of metastases, and, in the future, for detecting the disease recurrence, as well as for monitoring the course of the treatment.

Acknowledgements. The authors are deeply grateful to the staff of the Novosibirsk Regional Oncology Hospital for their assistance in patients' examination.

Research funding. The study was carried out within the program "Epidemiological monitoring of public health and studying the genetic and molecular biology mechanisms of the common internal diseases in Siberia aiming to improve the diagnosis, prevention, and treatment", state assignment No.0324-2018-0001, registration number AAAA-A17-117112850280-2.

Conflict of interest. The authors declare no conflicts of interest to be reported.

\section{References}

1. Fedorov V.E., Podelyakin K.A. Epidemiological aspects of colorectal cancer. Medicinskij al'manah 2017; 4: 145-148, https://doi.org/10.21145/2499-9954-2017-4-145-148.
2. Pathak S., Pandanaboyana S., Daniels I., Smart N., Prasad K.R. Obesity and colorectal liver metastases: mechanisms and management. Surg Oncol 2016; 25(3): 246251, https://doi.org/10.1016/j.suronc.2016.05.021.

3. Stagnitti A., Barchetti F., Barchetti G., Pasqualitto E., Sartori A., Glorioso M., Gigli S., Buonocore V., Monti M.L., Marini A., Mele C., Stagnitti F., Laghi A. Preoperative staging of colorectal cancer using virtual colonoscopy: correlation with surgical results. Eur Rev Med Pharmacol Sci 2015; 19(9): 1645-1651.

4. Meyerhardt J.A., Mangu P.B., Flynn P.J., Korde L., Loprinzi C.L., Minsky B.D., Petrelli N.J., Ryan K., Schrag D.H., Wong S.L., Benson A.B. $3^{\text {rd }}$; American Society of Clinical Oncology. Follow-up care, surveillance protocol, and secondary prevention measures for survivors of colorectal cancer: American Society of Clinical Oncology clinical practice guideline endorsement. J Clin Oncol 2013; 31(35): 4465-4470, https://doi.org/10.1200/jco.2013.50.7442.

5. Hamilton T.D., Leugner D., Kopciuk K., Dixon E., Sutherland F.R., Bathe O.F. Identification of prognostic inflammatory factors in colorectal liver metastases. BMC Cancer 2014; 14: 542, https://doi.org/10.1186/1471-240714-542.

6. Sasaki K., Andreatos N., Margonis G.A., He J., Weiss M., Johnston F., Wolfgang C., Antoniou E., Pikoulis E., Pawlik T.M. The prognostic implications of primary colorectal tumor location on recurrence and overall survival in patients undergoing resection for colorectal liver metastasis. J Surg Oncol 2016; 114(7): 803-809, https://doi.org/10.1002/ jso. 24425 .

7. Mavros M.N., Hyder O., Pulitano C., Aldrighetti L., Pawlik T.M. Survival of patients operated for colorectal liver metastases and concomitant extra-hepatic disease: external validation of a prognostic model. J Surg Oncol 2013; 107(5); 481-485, https://doi.org/10.1002/jso.23260.

8. Kim C.W., Kim J.I., Park S.H., Han J.Y., Kim J.K., Chung K.W., Sun H.S. Usefulness of plasma tumor M2pyruvate kinase in the diagnosis of gastrointestinal cancer. Korean J Gastroenterol 2003; 42(5): 387-393.

9. Dombrauckas J.D., Santarsiero B.D., Mesecar A.D. Structural basis for tumor pyruvate kinase M2 allosteric regulation and catalysis. Biochemistry 2005; 44(27): 94179429, https://doi.org/10.1021/bi0474923.

10. Zaccaro C., Saracino I.M., Fiorini G., Figura N., Holton J., Castelli V., Pesci V., Gatta L., Vaira D. Power of screening tests for colorectal cancer enhanced by high levels of M2-PK in addition to FOBT. Intern Emerg Med 2017; 12(3): 333-339, https://doi.org/10.1007/s11739-017-1610-3.

11. Guan M., Tong Y., Guan M., Liu X., Wang M., Niu R., Zhang F., Dong D., Shao J., Zhou Y. Lapatinib inhibits breast cancer cell proliferation by influencing PKM2 expression. Technol Cancer Res Treat 2018; 17: 1533034617749418, https://doi.org/10.1177/1533034617749418.

12. Tee S.S., Park J.M., Hurd R.E., Brimacombe K.R., Boxer M.B., Massoud T.F., Rutt B.K., Spielman D.M. PKM2 activation sensitizes cancer cells to growth inhibition by 2-deoxy-D-glucose. Oncotarget 2017; 8(53): 90959-90968, https://doi.org/10.18632/oncotarget.19630.

13. Krones A., Jungermann K., Kietzmann T. Crosstalk between the signals hypoxia and glucose at the glucose response element of the L-type pyruvate kinase gene. Endocrinology 2001; 142(6): 2707-2718, https://doi. org/10.1210/endo.142.6.8200. 


\section{CLINICAL SUPPLEMENTS}

14. Fung K.Y., Tabor B., Buckley M.J., Priebe I.K., Purins L., Pompeia C., Brierley G.V., Lockett T., Gibbs P., Tie J., McMurrick P., Moore J., Ruszkiewicz A., Nice E., Adams T.E., Burgess A., Cosgrove L.J. Blood-based protein biomarker panel for the detection of colorectal cancer. PLOS One 2015; 10(3): e0120425, https://doi.org/10.1371/journal. pone.0120425.

15. Jenkins C.A., Lewis P.D., Dunstan P.R., Harris D.A. Role of Raman spectroscopy and surface enhanced Raman spectroscopy in colorectal cancer. World J Gastrointest Oncol 2016; 8(5): 427-438, https://doi.org/10.4251/wjgo.v8.i5.427.

16. Sankiewicz A., Lukaszewski Z., Trojanowska K., Gorodkiewicz E. Determination of collagen type IV by Surface Plasmon Resonance Imaging using a specific biosensor. Anal Biochem 2016; 515: 40-46, https://doi.org/10.1016/j. ab.2016.10.002.

17. Jain R., Rawat A., Verma B., Markiewski M.M., Weidanz J.A. Antitumor activity of a monoclonal antibody targeting major histocompatibility complex class I-Her2 peptide complexes. J Natl Cancer Inst 2013; 105(3): 202-218, https:// doi.org/10.1093/jnci/djs521.

18. Kang Y.R., Byun J.S., Kim T.J., Park H.G., Park J.C., Barange N., Nam K.H., Kim Y.D. Monitoring of the binding between EGFR protein and EGFR aptamer using in-situ total internal reflection ellipsometry. J Nanosci Nanotechnol 2016; 16(6): 6445-6449, https://doi.org/10.1166/jnn.2016. 12123.

19. Loitsch S.M., Shastri Y., Stein J. Stool test for colorectal cancer screening — it's time to move! Clin Lab 2008; 54(1112): 473-484.

20. Jonkheijm P., Weinrich D., Schröder H., Niemeyer C.M., Waldmann $\mathrm{H}$. Chemical strategies for generating protein biochips. Angew Chem Int Ed Engl 2008; 47(50): 9618-9647, https://doi.org/10.1002/anie.200801711.

21. Rykhlitskiy S.V., Kruchinin V.N., Shvets V.A., Spesivtsev E.V., Prokop'ev V.Yu. Spectral plasmonellipsometric complex ELLIPS-SPEC. Pribory $i$ tekhnika eksperimenta 2013; 1: 137-138, https://doi.org/10.7868/ s0032816212060092.

22. Kikawada M., Ono A., Inami W., Kawata Y. Plasmonenhanced autofluorescence imaging of organelles in label-free cells by deep-ultraviolet excitation. Anal Chem 2016; 88(2): 1407-1411, https://doi.org/10.1021/acs. analchem.5b04060.

23. Handbook of ellipsometry. Tompkins H.G., Irene E.A. (editors). Springer Berlin Heidelberg; 2005; 789 p., https://doi. org/10.1007/3-540-27488-x.

24. Bye W.A., Nguyen T.M., Parker C.E., Jairath V., East J.E. Strategies for detecting colon cancer in patients with inflammatory bowel disease. Cochrane Database Syst Rev 2017; 9: CD000279, https://doi.org/10.1002/14651858. cd000279.pub4.

25. Chekulayev V., Mado K., Shevchuk I., Koit A., Kaldma A., Klepinin A., Timohhina N., Tepp K., Kandashvili M., Ounpuu L., Heck K., Truu L., Planken A., Valvere V., Kaambre T. Metabolic remodeling in human colorectal cancer and surrounding tissues: alterations in regulation of mitochondrial respiration and metabolic fluxes. Biochem Biophys Rep 2015; 4: 111-125, https://doi.org/10.1016/j.bbrep.2015.08.020.

26. Meng W., Zhu H.H., Xu Z.F., Cai S.R., Dong Q., Pan Q.R., Zheng S., Zhang S.Z. Serum M2-pyruvate kinase: a promising non-invasive biomarker for colorectal cancer mass screening. World J Gastrointest Oncol 2012; 4(6): 145-151, https://doi.org/10.4251/wjgo.v4.i6.145.

27. Abdullah M., Rani A.A., Simadibrata M., Fauzi A., Syam A.F. The value of fecal tumor M2 pyruvate kinase as a diagnostic tool for colorectal cancer screening. Acta Med Indones 2012; 44(2): 94-99.

28. Parente F., Marino B., Ilardo A., Fracasso P., Zullo A., Hassan C., Moretti R., Cremaschini M., Ardizzoia A., Saracino I., Perna F., Vaira D. A combination of faecal tests for the detection of colon cancer: a new strategy for an appropriate selection of referrals to colonoscopy? A prospective multicentre Italian study. Eur J Gastroenterol Hepatol 2012; 24(10): 1145-1152, https://doi.org/10.1097/ meg.0b013e328355cc79.

29. Haug U., Rothenbacher D., Wente M.N., Seiler C.M., Stegmaier C., Brenner H. Tumour M2-PK as a stool marker for colorectal cancer: comparative analysis in a large sample of unselected older adults vs colorectal cancer patients. $\mathrm{Br}$ J Cancer 2007; 96(9): 1329-1334, https://doi.org/10.1038/ sj.bjc. 6603712 . 